Sharif University of Technology
Scientia Iranica
Transactions E: Industrial Engineering
http://scientiairanica.sharif.edu
IRAN ICA

\title{
Hartley-Ross type unbiased estimators of population mean using two auxiliary variables
}

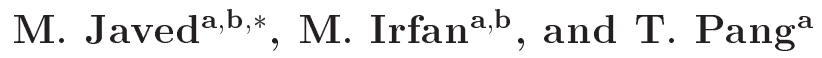 \\ a. Department of Mathematics, Institute of Statistics, Zhejiang University, Hangzhou 310027, China. \\ b. Department of Statistics, Government College University, Faisalabad, Pakistan. \\ Received 26 November 2017; received in revised form 10 May 2018; accepted 21 July 2018
}

\section{KEYWORDS}

Auxiliary variable;

Hartley-Ross type

estimator;

Unbiased;

Variance.

\begin{abstract}
In survey sampling, it is a well-established phenomenon that the efficiency of estimators increases with proper information on auxiliary variable(s). Keeping this fact in mind, the information on two auxiliary variables was utilized to propose a family of Hartley-Ross type unbiased estimators for estimating population mean under simple random sampling without replacement. Minimum variance of the new estimators was derived up to the first degree of approximation. Three real datasets were used to verify the efficient performance of the new family in comparison to the usual unbiased, Hartley and Ross, and other competing estimators.
\end{abstract}

(C) 2019 Sharif University of Technology. All rights reserved.

\section{Introduction}

In the process of conducting sample surveys, researchers normally use ratio-type estimators when there is a need to estimate the unknown population parameters of the study variable by means of known population parameters of a correlated auxiliary variable(s). An eye view of literature on the estimation of population mean under simple random sampling without replacement (SRSWOR) with proper information on two auxiliary variables shows prominent studies of AbuDayyeh et al. [1], Kadilar and Cingi [2], Singh and Tailor [3], Lu and Yan [4], Lu et al. [5], Vishwakarma and Kumar [6], Sharma and Singh [7], Yasmeen et al. [8], Lu [9], Muneer et al. [10], and Shabbir and Gupta [11].

One eminent disadvantage of using ratio-type es-

\footnotetext{
* Corresponding author. Tel.: +86 13250808280 E-mail addresses: mariastat@zju.edu.cn (M. Javed); mirfan@zju.edu.cn (M. Irfan); txpang@zju.edu.cn (T. Pang).
}

doi: $10.24200 /$ sci. 2018.5648 .1397 timators is that they are typically biased. Hartely and Ross [12] initiated the concept of unbiased estimators for estimating population mean. Similar efforts for unbiased estimators were carried out by Robson [13], Murthy and Nanjamma [14], Biradar and Singh [1517], Sahoo et al. [18], Singh et al. [19], Cekim and Kadilar [20], and Khan et al. [21]. Applying different sampling techniques, they used the information on a single auxiliary variable. Continuing these efforts, we proposed a new optimal family of Hartley-Ross type unbiased estimators with the novelty that the information on two auxiliary variables is used.

Consider $\Theta=\left\{\Theta_{1}, \Theta_{2}, \Theta_{3}, \cdots, \Theta_{N}\right\}$ as $N$ units of a finite population. Let the $i$ th unit of population, $y_{i}$, be the value of study variable $y$ and $x_{i}, z_{i}$ be the values of auxiliary variables $x$ and $z$, respectively. $n$ is the size of a sample selected from the population under SRSWOR scheme given that $n<N$. Some usual measures related to the study variable and auxiliary variables are presented in Table 1.

The following relative error terms are used to derive the expressions for the bias, variance, and minimum variance of the existing and suggested estimators: 
Table 1. Measures associated with study variable $(y)$ and auxiliary variables $(x$ and $z)$.

\begin{tabular}{lccc}
\hline \multicolumn{1}{c}{ Measure } & Study variable $\boldsymbol{y}$ & Auxiliary variable $\boldsymbol{x}$ & Auxiliary variable $\boldsymbol{z}$ \\
\hline Population mean & $\bar{Y}=N^{-1} \sum_{i=1}^{N} y_{i}$ & $\bar{X}=N^{-1} \sum_{i=1}^{N} x_{i}$ & $\bar{Z}=N^{-1} \sum_{i=1}^{N} z_{i}$ \\
Sample mean & $\bar{y}=n^{-1} \sum_{i=1}^{n} y_{i}$ & $\bar{x}=n^{-1} \sum_{i=1}^{n} x_{i}$ & $\bar{z}=n^{-1} \sum_{i=1}^{n} z_{i}$ \\
Population variance & $S_{y}^{2}=\frac{\sum_{i=1}^{N}\left(y_{i}-\bar{Y}\right)^{2}}{(N-1)}$ & $S_{x}^{2}=\frac{\sum_{i=1}^{N}\left(x_{i}-\bar{X}\right)^{2}}{(N-1)}$ & $S_{z}^{2}=\frac{\sum_{i=1}^{N}\left(z_{i}-\bar{Z}\right)^{2}}{(N-1)}$ \\
Coefficient of variation & $C_{y}=\bar{Y}^{-1} S_{y}$ & $C_{x}=\bar{X}^{-1} S_{x}$ & $C_{z}=\bar{Z}^{-1} S_{z}$ \\
\hline
\end{tabular}

Table 2. Measures used in relative error terms.

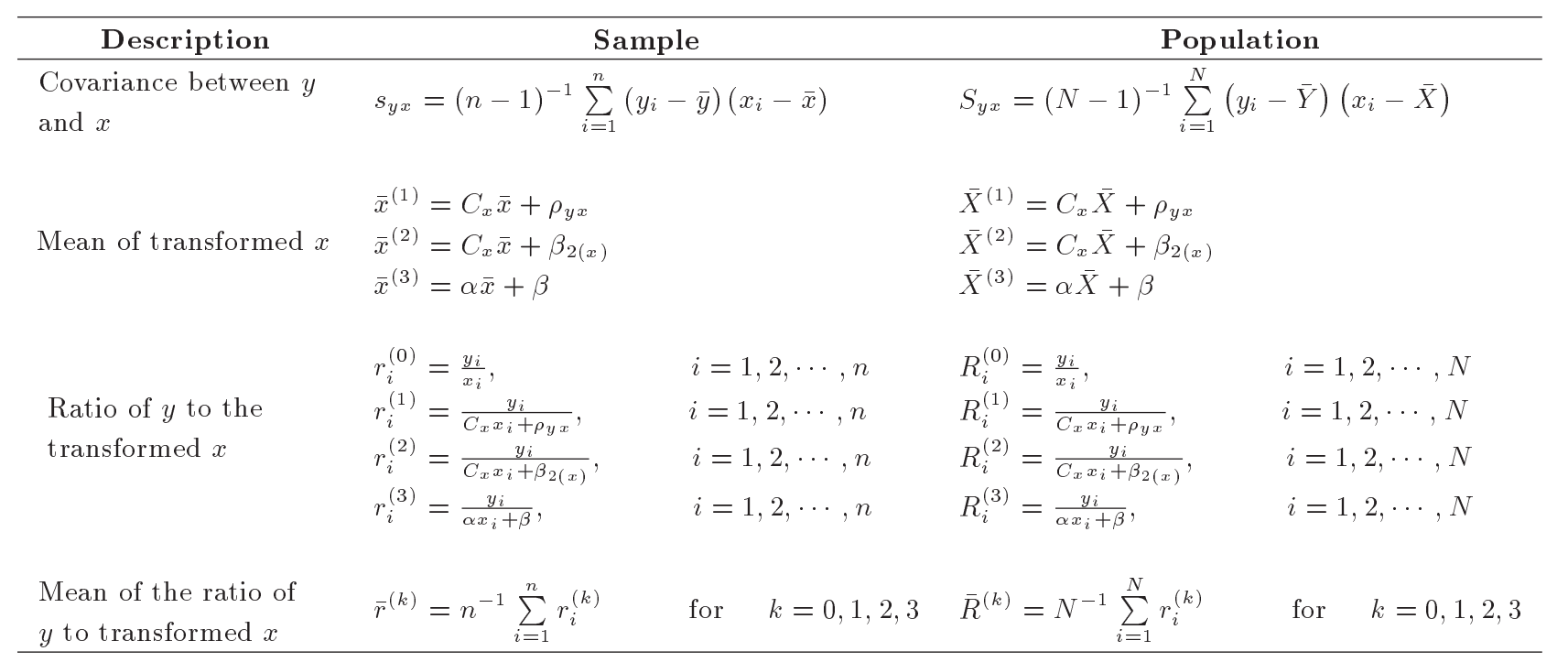

$$
\begin{array}{ll}
\varepsilon_{0}=\frac{\bar{y}}{\bar{Y}}-1, & \varepsilon_{1}=\frac{\bar{x}}{\bar{X}}-1, \\
\varepsilon_{2}=\frac{\bar{z}}{\bar{Z}}-1, & \varepsilon_{3}=\frac{s_{y x}}{S_{y x}}-1, \\
\varepsilon_{4}=\frac{\bar{x}^{(j)}}{\bar{X}^{(j)}}-1, & \text { for } j=1,2,3, \\
\varepsilon_{5}=\frac{\bar{r}^{(k)}}{\bar{R}^{(k)}}-1, & \text { for } k=0,1,2,3 .
\end{array}
$$

Table 2 presents a detailed description of some terms used in Eq. (1).

Remark 1.1. $s_{y x}, \bar{x}^{(j)}$, and $\bar{r}^{(k)}$ are the unbiased estimators of their population parameters $S_{y x}, \bar{X}^{(j)}$, and $\bar{R}^{(k)}$, respectively.

Remark 1.2. The expectations of the relative errors given in Eq. (1) are as follows [19,20]:

$$
\begin{array}{ll}
E\left(\varepsilon_{i}\right)=0, & \text { for } i=0,1,2,3,4,5, \\
E\left(\varepsilon_{0}^{2}\right)=\psi C_{y}^{2}, & E\left(\varepsilon_{1}^{2}\right)=\psi C_{x}^{2}, \\
E\left(\varepsilon_{2}^{2}\right)=\psi C_{z}^{2}, & E\left(\varepsilon_{3}^{2}\right)=\psi\left(\frac{\theta_{22 x}}{\rho_{y x}}-1\right), \\
E\left(\varepsilon_{4}^{2}\right)=\psi C_{x^{(j)}}^{2}, & E\left(\varepsilon_{5}^{2}\right)=\psi C_{r^{(k)}}^{2},
\end{array}
$$

$$
\begin{aligned}
& E\left(\varepsilon_{0} \varepsilon_{1}\right)=\psi \rho_{y x} C_{y} C_{x}=\psi C_{y x} \\
& E\left(\varepsilon_{0} \varepsilon_{2}\right)=\psi \rho_{y z} C_{y} C_{z}=\psi C_{y z}, \\
& E\left(\varepsilon_{0} \varepsilon_{3}\right)=\psi\left(\frac{C_{y} \theta_{21 x}}{\rho_{y x}}\right) \\
& E\left(\varepsilon_{1} \varepsilon_{2}\right)=\psi \rho_{x z} C_{x} C_{z}=\psi C_{x z} \\
& E\left(\varepsilon_{1} \varepsilon_{3}\right)=\psi\left(\frac{C_{x} \theta_{12 x}}{\rho_{y x}}\right) \\
& E\left(\varepsilon_{2} \varepsilon_{3}\right)=\psi\left(\frac{C_{z} \theta_{12 z}}{\rho_{y z}}\right)
\end{aligned}
$$

where:

$$
\psi=\left(\frac{1}{n}-\frac{1}{N}\right)
$$

is finite population correction factor;

$$
C_{x}^{(j)}=\left(\bar{X}^{(j)}\right)^{-1} S_{x^{(j)}}
$$

is coefficient of variation for transformed $x$;

$$
C_{r}^{(k)}=\left(\bar{R}^{(k)}\right)^{-1} S_{r^{(k)}}
$$

is coefficient of variation for ratio of $y$ to transformed $x$; 


$$
S_{x^{(j)}}=\sqrt{(N-1)^{-1} \sum_{i=1}^{N}\left(x_{i}^{(j)}-\bar{X}^{(j)}\right)^{2}}
$$

is standard deviation of transformed $x$

$$
S_{r^{(k)}}=\sqrt{(N-1)^{-1} \sum_{i=1}^{N}\left(r_{i}^{(k)}-\bar{R}^{(k)}\right)^{2}}
$$

is standard deviation of ratio of $y$ to transformed $x$;

$$
S_{y x^{(j)}}=\sqrt{(N-1)^{-1} \sum_{i=1}^{N}\left(y_{i}-\bar{Y}\right)\left(x_{i}^{(j)}-\bar{X}^{(j)}\right)}
$$

is covariance between $y$ and transformed $x$ (for all above expressions $j=1,2,3$ and $k=0,1,2,3$ );

$$
\begin{aligned}
& \rho_{y x}=\left(S_{y} S_{x}\right)^{-1} S_{y x} \\
& \rho_{y z}=\left(S_{y} S_{z}\right)^{-1} S_{y z} \\
& \rho_{x z}=\left(S_{x} S_{z}\right)^{-1} S_{x z}
\end{aligned}
$$

are correlation coefficients between $y \& x, y \& z$, and $x \& z$, respectively;

$$
\begin{aligned}
& S_{y x}=(N-1)^{-1} \sum_{i=1}^{N}\left(y_{i}-\bar{Y}\right)\left(x_{i}-\bar{X}\right) \\
& S_{y z}=(N-1)^{-1} \sum_{i=1}^{N}\left(y_{i}-\bar{Y}\right)\left(z_{i}-\bar{Z}\right) \\
& S_{x z}=(N-1)^{-1} \sum_{i=1}^{N}\left(x_{i}-\bar{X}\right)\left(z_{i}-\bar{Z}\right)
\end{aligned}
$$

are covariances between $y \& x, y \& z$, and $x \& z$, respectively.

Values of $\theta_{21 x}, \theta_{12 x}, \theta_{22 x}$, and $\theta_{12 z}$ can be obtained through the following expressions:

$$
\begin{aligned}
& \theta_{p q x}=\frac{\mu_{p q x}}{\left(\mu_{20 x}^{\frac{p}{2}}\right)\left(\mu_{02 x}^{\frac{q}{2}}\right)}, \quad \mu_{p q x}=\frac{\sum_{i=1}^{N}\left(y_{i}-\bar{Y}\right)^{p}\left(x_{i}-\bar{X}\right)^{q}}{N}, \\
& \theta_{p q z}=\frac{\mu_{p q z}}{\left(\mu_{20 z}^{\frac{p}{2}}\right)\left(\mu_{02 z}^{\frac{q}{2}}\right)}, \quad \mu_{p q z}=\frac{\sum_{i=1}^{N}\left(y_{i}-\bar{Y}\right)^{p}\left(z_{i}-\bar{Z}\right)^{q}}{N},
\end{aligned}
$$

for $p, q=0,1,2$.

\section{Review of literature}

This section presents some estimators from the literature when estimating population mean under simple random sampling.
- The traditional unbiased estimator for unknown population mean along with its variance is:

$$
\begin{aligned}
& \bar{y}_{0}^{(u)}=\bar{y}, \\
& \operatorname{Var}\left(\bar{y}_{0}^{(u)}\right)=\psi \bar{Y}^{2} C_{y}^{2} .
\end{aligned}
$$

- Hartley and Ross [12] initiated an unbiased ratiotype estimator as given below:

$$
\bar{y}_{H R}^{(u)}=\bar{r}^{(0)} \bar{X}+\frac{n(N-1)}{N(n-1)}\left(\bar{y}-\bar{r}^{(0)} \bar{x}\right),
$$

where:

$$
\bar{r}^{(0)}=n^{-1} \sum_{i=1}^{n} r_{i}^{(0)}, \quad r_{i}^{(0)}=\frac{y_{i}}{x_{i}} .
$$

The variance of this estimator in the first order of approximation is equal to the mean square error of the usual ratio estimator (see Singh and Mangat [22]).

$$
\operatorname{Var}\left(\bar{y}_{H R}^{(u)}\right) \cong \psi \bar{Y}^{2}\left[C_{y}^{2}+C_{x}^{2}-2 \rho_{y x} C_{y} C_{x}\right] .
$$

- Grover and Kaur [23] followed the lines of Gupta and Shabbir [24] and Shabbir and Gupta [25] and suggested a generalized class based on ratio-type exponential estimators as follows:

$$
\bar{y}_{G K}=\left[q_{1} \bar{y}+q_{2}(\bar{X}-\bar{x})\right] \exp \left[\frac{\alpha(\bar{X}-\bar{x})}{\alpha(\bar{X}+\bar{x})+2 \beta}\right],
$$

where $q_{1}$ and $q_{2}$ are the suitable weights to be chosen. $\alpha(\neq 0)$ and $\beta$ are either known quantities or functions of any known population parameters, including coefficient of variation, coefficient of skewness, coefficient of Kurtosis, coefficient of correlation, etc. Box I.

The optimal values of $q_{1}$ and $q_{2}$ are shown in

We get the minimum MSE by utilizing the optimal values shown in Box I. The expression for minimum MSE up to the first degree of approximation is shown in Box II.

- Singh et al. [19] suggested two Hartley-Ross type estimators by considering the estimators of Kadilar and Cingi [26] and Upadhyaya and Singh [27]:

$$
\begin{aligned}
& \bar{y}_{S 1}^{(u)}=\bar{r}^{(1)} \bar{X}^{(1)}+\frac{n(N-1)}{N(n-1)}\left(\bar{y}-\bar{r}^{(1)} \bar{x}^{(1)}\right), \\
& \bar{y}_{S 2}^{(u)}=\bar{r}^{(2)} \bar{X}^{(2)}+\frac{n(N-1)}{N(n-1)}\left(\bar{y}-\bar{r}^{(2)} \bar{x}^{(2)}\right),
\end{aligned}
$$

where:

$$
\bar{r}^{(1)}=n^{-1} \sum_{i=1}^{n} r_{i}^{(1)}, \quad r_{i}^{(1)}=\frac{y_{i}}{C_{x} x_{i}+\rho_{y x}}=\frac{y_{i}}{x_{i}^{(1)}},
$$




$$
\begin{aligned}
q_{1(o p t)} & =\frac{8-\psi \lambda^{2} C_{x}^{2}}{8\left[1+\psi C_{y}^{2}\left(1-\rho_{y x}^{2}\right)\right]}, \\
q_{2(o p t)} & =\frac{\bar{Y}\left[\psi \lambda^{3} C_{x}^{3}+8 C_{y} \rho_{y x}-\psi \lambda^{2} C_{x}^{2} C_{y} \rho_{y x}-4 \lambda C_{x}\left\{1-\psi C_{y}^{2}\left(1-\rho_{y x}^{2}\right)\right\}\right]}{8 \bar{X} C_{x}\left[1+\psi C_{y}^{2}\left(1-\rho_{y x}^{2}\right)\right]},
\end{aligned}
$$

where:

$$
\lambda=\frac{\alpha \bar{X}}{\alpha \bar{X}+\beta} .
$$

\section{Box I}

$$
M S E_{\min }\left(\bar{y}_{G K}\right) \cong \frac{\psi \bar{Y}^{2}\left[64 C_{y}^{2}\left(1-\rho_{y x}^{2}\right)-\psi \lambda^{4} C_{x}^{4}-16 \psi \lambda^{2} C_{x}^{2} C_{y}^{2}\left(1-\rho_{y x}^{2}\right)\right]}{64\left[1+\psi C_{y}^{2}\left(1-\rho_{y x}^{2}\right)\right]}
$$

Box II

$$
\begin{aligned}
& \bar{X}^{(1)}=C_{x} \bar{X}+\rho_{y x}, \\
& \bar{r}^{(2)}=n^{-1} \sum_{i=1}^{n} r_{i}^{(2)}, \quad r_{i}^{(2)}=\frac{y_{i}}{C_{x} x_{i}+\beta_{2(x)}}=\frac{y_{i}}{x_{i}^{(2)}}, \\
& \bar{X}^{(2)}=C_{x} \bar{X}+\beta_{2(x)},
\end{aligned}
$$

where $\rho_{y x}$ is the coefficient of correlation between study variable $y$ and auxiliary variable $x$, and $\beta_{2(x)}$ is the coefficient of kurtosis of auxiliary variable $x$.

Given below are the variances of $\bar{y}_{S 1}^{(u)}$ and $\bar{y}_{S 2}^{(u)}$ :

$\operatorname{Var}\left(\bar{y}_{S 1}^{(u)}\right) \cong \psi\left[S_{y}^{2}+\left(\bar{R}^{(1)} S_{x^{(1)}}\right)^{2}-2 \bar{R}^{(1)} S_{y x^{(1)}}\right]$

$\operatorname{Var}\left(\bar{y}_{S 2}^{(u)}\right) \cong \psi\left[S_{y}^{2}+\left(\bar{R}^{(2)} S_{x^{(2)}}\right)^{2}-2 \bar{R}^{(2)} S_{y x^{(2)}}\right]$

where $\bar{R}^{(1)}$ and $\bar{R}^{(2)}$ are defined in Table 2 , and $S_{x^{(1)}}$, $S_{x^{(2)}}, S_{y x^{(1)}}$, and $S_{y x^{(2)}}$ are defined in Remark 1.2.

- A special version of estimators of Khoshnevisan et al. [28] was utilized by Cekim and Kadilar [20] to form a general family of Hartley-Ross type unbiased estimators as follows:

$$
\bar{y}_{C K 1}^{(u)}=\bar{r}^{(3)} \bar{X}^{(3)}+\frac{n(N-1)}{N(n-1)}\left(\bar{y}-\bar{r}^{(3)} \bar{x}^{(3)}\right),
$$

where:

$$
\begin{aligned}
& \bar{r}^{(3)}=n^{-1} \sum_{i=1}^{n} r_{i}^{(3)}, \quad r_{i}^{(3)}=\frac{y_{i}}{\alpha x_{i}+\beta}=\frac{y_{i}}{x_{i}^{(3)}}, \\
& \bar{X}^{(3)}=\alpha \bar{X}+\beta .
\end{aligned}
$$

$\alpha$ and $\beta$ are explained earlier.

The variance of $\bar{y}_{C K 1}^{(u)}$ is given by:

$\operatorname{Var}\left(\bar{y}_{C K 1}^{(u)}\right) \cong \psi\left[S_{y}^{2}+\left(\bar{R}^{(3)} S_{x^{(3)}}\right)^{2}-2 \bar{R}^{(3)} S_{y x(3)}\right]$

where $\bar{R}^{(3)}$ is defined in Table 2 , and $S_{x^{(3)}}$ and $S_{y x^{(3)}}$ are defined in Remark 1.2.

Remark 2.1. Some important considerations can be noticed here. If we have:

$$
\begin{aligned}
& \text { i) } \quad \alpha=C_{x} \quad \text { and } \quad \beta=\rho_{y x} \quad \text { in } r_{i}^{(3)} \text {, then } \\
& r_{i}^{(3)}=r_{i}^{(1)} \quad \text { and } \quad \bar{y}_{C K 1}^{(u)}=\bar{y}_{S 1}^{(u)} \text {; } \\
& \text { ii) } \alpha=C_{x} \quad \text { and } \quad \beta=\beta_{2(x)} \quad \text { in } \quad r_{i}^{(3)} \text {, then } \\
& r_{i}^{(3)}=r_{i}^{(2)} \quad \text { and } \quad \bar{y}_{C K 1}^{(u)}=\bar{y}_{S 2}^{(u)} \text {. }
\end{aligned}
$$

- Another family of Hartley-Ross type unbiased estimators from a special version of Koyuncu and Kadilar [29] was proposed by Cekim and Kadilar [20] and is defined as follows:

$$
\begin{aligned}
\bar{y}_{C K 2}^{(u)}= & q_{3} \bar{y}\left[\frac{\alpha \bar{X}+\beta}{\gamma(\alpha \bar{x}+\beta)+(1-\gamma)(\alpha \bar{X}+\beta)}\right]^{t} \\
& -q_{3} \bar{y} \psi\left[\frac{t(t+1)}{2} \gamma^{2} \lambda^{2} C_{x}^{2}-t \gamma \lambda \frac{s_{y x}}{\bar{y} \bar{X}}\right] \\
& -\left(q_{3}-1\right) \bar{y},
\end{aligned}
$$


where $q_{3}$ is the suitable weight that minimizes the variance, $t=1, \gamma=1, \lambda=\frac{\alpha \bar{X}}{\alpha \bar{X}+\beta}$, and $\alpha$ and $\beta$ were defined earlier.

$$
\begin{aligned}
\operatorname{Var} & \left(\bar{y}_{C K 2}^{(u)}\right) \cong \bar{Y}^{2} \psi\left[\left\{q_{3}^{2} t^{2} \gamma^{2} \lambda^{2} C_{x}^{2}-2 q_{3} t \gamma \lambda C_{y x}+C_{y}^{2}\right\}\right. \\
& -\psi\left\{q_{3} t \gamma \lambda\left(\frac{t+1}{2} \gamma \lambda C_{x}^{2}-C_{y x}\right)\right\}^{2} \\
& -\psi q_{3} t \gamma \lambda\left\{2 \frac{C_{y x}}{\rho_{y x}}\left(q_{3} t \gamma \lambda C_{x} \theta_{12 x}-C_{y} \theta_{21 x}\right)\right. \\
& \left.\left.-(t+1) \gamma \lambda C_{x}^{2}\left(q_{3} t \gamma \lambda C_{y x}-C_{y}^{2}\right)\right\}\right]
\end{aligned}
$$

The optimal value of $q_{3}$ is given below:

$$
q_{3(o p t)}=\frac{\Psi}{\Delta}
$$

where:

$$
\begin{aligned}
\Psi= & t \gamma \lambda\left[C_{y x}\left\{1-\frac{\psi C_{y} \theta_{21 x}}{\rho_{y x}}\right\}+\frac{(t+1)}{2} \psi \gamma \lambda C_{x}^{2} C_{y}^{2}\right] \\
\Delta= & t^{2} \gamma^{2} \lambda^{2}\left[C_{x}^{2}+\psi\left\{C_{y x}\left((t+1) \gamma \lambda C_{x}^{2}-2 \frac{C_{x} \theta_{12 x}}{\rho_{y x}}\right)\right.\right. \\
& \left.\left.-\left(\frac{(t+1)}{2} \gamma \lambda C_{x}^{2}-C_{y x}\right)^{2}\right\}\right]
\end{aligned}
$$

We have the minimum variance by placing the optimal value of $q_{3}$ in Eq. (15) as follows:

$$
\operatorname{Var}_{\min }\left(\bar{y}_{C K 2}^{(u)}\right) \cong \bar{Y}^{2} \psi\left(C_{y}^{2}-\frac{\Psi^{2}}{\Delta}\right)
$$

- Muneer et al. [10] followed the lines of Gupta and Shabbir [24] and Singh and Singh [30] to propose the following estimators. These estimators utilized the information on two auxiliary variables.

$$
\begin{aligned}
\bar{y}_{M U, \gamma}= & {\left[q_{4} \bar{y}+q_{5}(\bar{X}-\bar{x})\right]\left[\gamma\left\{2-\exp \left(\frac{\bar{z}-\bar{Z}}{\bar{z}+\bar{Z}}\right)\right\}\right.} \\
& \left.+(1-\gamma) \exp \left(\frac{\bar{Z}-\bar{z}}{\bar{Z}+\bar{z}}\right)\right] .
\end{aligned}
$$

By putting $\gamma=0$ and 1 , two estimators $\bar{y}_{M U, 0}$ and $\bar{y}_{M U, 1}$ are obtained; $q_{4}$ and $q_{5}$ are the constants to be determined.

The optimal values of $q_{4}$ and $q_{5}$ are shown in Box III. Using $q_{4(o p t)}$ and $q_{5(o p t)}$, we get the $M S E_{\min }\left(\bar{y}_{M U, \gamma}\right)$ as shown in Box IV.

- Recently, Shabbir and Gupta [11] used the linear combination of two auxiliary variables to propose a difference cum exponential ratio-type estimator. This concept was initiated by Gupta and Shabbir [24] and Grover and Kaur [31].

$$
\bar{y}_{S G}=\left[q_{6} \bar{y}+q_{7}(\bar{X}-\bar{x})+q_{8}(\bar{Z}-\bar{z})\right] \exp \left(\frac{\bar{X}-\bar{x}}{\bar{X}+\bar{x}}\right)_{(19}
$$

where $q_{6}, q_{7}$, and $q_{8}$ are the feasible constants defined in Box V.

The minimum MSE is obtained using the above optimal constants as follows:

$$
\begin{aligned}
& M S E_{\min }\left(\bar{y}_{S G}\right) \cong \bar{Y}^{2}[1 \\
& \left.\quad-\frac{\left(1+\frac{1}{64} \psi^{2} C_{x}^{4}\right)+\frac{1}{4} \psi^{2} C_{y}^{2} C_{x}^{2}\left(1-R_{y \cdot x z}^{2}\right)}{1+\psi C_{y}^{2}\left(1-R_{y \cdot x z}^{2}\right)}\right]
\end{aligned}
$$

$$
\begin{aligned}
q_{4(o p t)} & =\frac{1+\left(\frac{3}{8}-\frac{\gamma}{4}\right) \psi C_{z}^{2}-\frac{1}{2} \psi C_{y z}-\frac{\psi C_{x z}\left(C_{x z}-C_{y x}\right)}{2 C_{x}^{2}}}{1+\psi C_{y}^{2}+\left(1-\frac{\gamma}{2}\right) \psi C_{z}^{2}-2 \psi C_{y z}-\frac{\psi\left(C_{x z}-C_{y x}\right)^{2}}{C_{x}^{2}}} \\
q_{5(o p t)} & =\bar{Y} \\
\bar{X} & {\left[\frac{C_{x z}}{2 C_{x}^{2}}-\frac{1+\left(\frac{3}{8}-\frac{\gamma}{4}\right) \psi C_{z}^{2}-\frac{1}{2} \psi C_{y z}-\frac{\psi C_{x z}\left(C_{x z}-C_{y x}\right)}{2 C_{x}^{2}}}{1+\psi C_{y}^{2}+\left(1-\frac{\gamma}{2}\right) \psi C_{z}^{2}-2 \psi C_{y z}-\frac{\psi\left(C_{x z}-C_{y x}\right)^{2}}{C_{x}^{2}}}\left\{\frac{C_{x z}-C_{y x}}{C_{x}^{2}}\right\}\right] . }
\end{aligned}
$$

\section{Box III}

$$
M S E_{\min }\left(\bar{y}_{M U, \gamma}\right) \cong \bar{Y}^{2}\left[1-\frac{\psi C_{x z}^{2}}{4 C_{x}^{2}}-\frac{\left\{1+\left(\frac{3}{8}-\frac{\gamma}{4}\right) \psi C_{z}^{2}-\frac{1}{2} \psi C_{y z}-\frac{\psi C_{x z}\left(C_{x z}-C_{y x}\right)}{2 C_{x}^{2}}\right\}^{2}}{1+\psi C_{y}^{2}+\left(1-\frac{\gamma}{2}\right) \psi C_{z}^{2}-2 \psi C_{y z}-\frac{\psi\left(C_{x z}-C_{y x}\right)^{2}}{C_{x}^{2}}}\right]
$$




$$
\begin{aligned}
q_{6(o p t)} & =\frac{1-\frac{1}{8} \psi C_{x}^{2}}{1+\psi C_{y}^{2}\left(1-R_{y . x z}^{2}\right)}, \\
q_{7(o p t)} & =\frac{\bar{Y}}{\bar{X}}\left[\frac{\frac{1}{2} C_{x}\left(1-\rho_{x z}^{2}\right)\left\{\psi C_{y}^{2}\left(1-R_{y . x z}^{2}\right)-\left(1-\frac{1}{4} \psi C_{x}^{2}\right)\right\}+C_{y}\left(\rho_{y x}-\rho_{y z} \rho_{x z}\right)\left(1-\frac{1}{8} \psi C_{x}^{2}\right)}{C_{x}\left(1-\rho_{x z}^{2}\right)\left\{1+\psi C_{y}^{2}\left(1-R_{y . x z}^{2}\right)\right\}}\right], \\
q_{8(o p t)} & =\frac{\bar{Y}}{\bar{Z}}\left[\frac{C_{y}\left(\rho_{y z}-\rho_{y x} \rho_{x z}\right)\left(1-\frac{1}{8} \psi C_{x}^{2}\right)}{C_{x}\left(1-\rho_{x z}^{2}\right)\left\{1+\psi C_{y}^{2}\left(1-R_{y . x z}^{2}\right)\right\}}\right],
\end{aligned}
$$

where $R_{y . x z}^{2}=\frac{\rho_{y x}^{2}+\rho_{y z}^{2}-2 \rho_{y x} \rho_{y z} \rho_{x z}}{1-\rho_{x z}^{2}}$.

\section{Box V}

\section{Proposed family of estimators}

It is valuable to mention that the estimator $\bar{y}_{S G}$ has a limited application due to the restricted transformation of the auxiliary variable. This section considers a general linear transformation of auxiliary information and uses a special version of Shabbir and Gupta [11] estimator to present a family of Hartley-Ross-type unbiased estimators. Minimum variance of the new class is also derived up to the first order of approximation.

Using a general linear transformation of auxiliary information in the Shabbir and Gupta [11] estimator, we get:

$$
\begin{aligned}
\left(\bar{y}_{S G, G e n}\right)= & {\left[q_{9} \bar{y}+q_{10}(\bar{X}-\bar{x})+q_{11}(\bar{Z}-\bar{z})\right] } \\
& \exp \left[\frac{\alpha(\bar{X}-\bar{x})}{\alpha(\bar{X}+\bar{x})+2 \beta}\right] .
\end{aligned}
$$

Bias, up to the first degree of approximation, of the generalized family presented in Eq. (21) is obtained as follows:

$$
\begin{aligned}
\operatorname{Bias} & \left(\bar{y}_{S G, G e n}\right) \cong\left(q_{9}-1\right) \bar{Y} \\
& +q_{9} \psi \bar{Y}\left(\frac{3}{8} \lambda^{2} C_{x}^{2}-\frac{1}{2} \lambda C_{x} C_{y} \rho_{y x}\right) \\
& +\frac{1}{2} q_{10} \psi \lambda \bar{X} C_{x}^{2}+\frac{1}{2} q_{11} \psi \lambda \bar{Z} C_{x} C_{z} \rho_{x z} .
\end{aligned}
$$

Subtracting $\operatorname{Bias}\left(\bar{y}_{S G, G e n}\right)$ from Eq. (21), we get:

$$
\begin{aligned}
& {\left[q_{9} \bar{y}+q_{10}(\bar{X}-\bar{x})+q_{11}(\bar{Z}-\bar{z})\right] \exp \left[\frac{\alpha(\bar{X}-\bar{x})}{\alpha(\bar{X}+\bar{x})+2 \beta}\right]} \\
& -\left[\left(q_{9}-1\right) \bar{Y}+q_{9} \psi \bar{Y}\left(\frac{3}{8} \lambda^{2} C_{x}^{2}-\frac{1}{2} \lambda \frac{S_{y x}}{\bar{X} \bar{Y}}\right)\right. \\
& \left.+\frac{1}{2} q_{10} \psi \lambda \bar{X} C_{x}^{2}+\frac{1}{2} q_{11} \psi \lambda \bar{Z} C_{x} C_{z} \rho_{x z}\right] .
\end{aligned}
$$

After doing some simplifications and replacing the parameters $\bar{Y}$ and $S_{y x}$ by their unbiased estimators $\bar{y}$ and $s_{y x}$ in Eq. (22), a new family of Hartley-Ross type unbiased estimators is proposed as follows:

$$
\begin{aligned}
\bar{y}_{P}^{(u)}= & {\left[q_{9} \bar{y}+q_{10}(\bar{X}-\bar{x})+q_{11}(\bar{Z}-\bar{z})\right] } \\
& \exp \left[\frac{\alpha(\bar{X}-\bar{x})}{\alpha(\bar{X}+\bar{x})+2 \beta}\right]+\bar{y}-\frac{1}{2} q_{10} \psi \lambda \bar{X} C_{x}^{2} \\
& -\frac{1}{2} q_{11} \psi \lambda C_{x z} \bar{Z}-q_{9} \bar{y}-\frac{3}{8} q_{9} \psi \lambda^{2} \bar{y} C_{x}^{2} \\
& +\frac{1}{2} q_{9} \psi \lambda \frac{s_{y x}}{\bar{X}},
\end{aligned}
$$

where $q_{9}, q_{10}$, and $q_{11}$ are the suitable weights to be chosen. $\alpha(\neq 0)$ and $\beta$ are either known constants or functions of any known population parameters, including coefficient of variation $C_{x}$ or $C_{z}$, coefficient of skewness $\beta_{1(x)}$, coefficient of Kurtosis $\beta_{2(x)}$, coefficient of correlation $\rho_{y x}$ or $\rho_{y z}$, etc., and $s_{y x}$ is an unbiased estimator of $S_{y x}$.

To express Eq. (23) in terms of $\varepsilon^{\prime} s$, the relative error terms defined in Eq. (1) are used. Expression is expanded up to the first order of approximation.

$$
\begin{aligned}
\bar{y}_{P}^{(u)} \cong & {\left[q_{9} \bar{Y}\left(1+\varepsilon_{0}\right)-q_{10} \bar{X} \varepsilon_{1}-q_{11} \bar{Z} \varepsilon_{2}\right] } \\
& {\left[1-\frac{1}{2} \lambda \varepsilon_{1}+\frac{3}{8} \lambda^{2} \varepsilon_{1}^{2}\right]+\bar{Y}\left(1+\varepsilon_{0}\right) } \\
& -\frac{1}{2} q_{10} \psi \lambda \bar{X} C_{x}^{2}-\frac{1}{2} q_{11} \psi \lambda C_{x z} \bar{Z}-q_{9} \bar{Y}\left(1+\varepsilon_{0}\right) \\
& -\frac{3}{8} q_{9} \psi \lambda^{2} C_{x}^{2} \bar{Y}\left(1+\varepsilon_{0}\right) \\
& +\frac{1}{2} q_{9} \psi \lambda \frac{S_{y x}\left(1+\varepsilon_{3}\right)}{\bar{X}} .
\end{aligned}
$$

Solving the above, we have: 


$$
\begin{aligned}
\left(\bar{y}_{P}^{(u)}-\bar{Y}\right) \cong & \bar{Y}\left(1-\frac{3}{8} q_{9} \psi \lambda^{2} C_{x}^{2}\right) \varepsilon_{0} \\
& -\left(\frac{1}{2} q_{9} \bar{Y} \lambda+q_{10} \bar{X}\right) \varepsilon_{1}-q_{11} \bar{Z} \varepsilon_{2} \\
& +\frac{1}{2} q_{9} \psi \lambda \frac{S_{y x}}{\bar{X}} \varepsilon_{3}-\frac{1}{2} q_{9} \bar{Y} \lambda \varepsilon_{0} \varepsilon_{1} \\
& +\frac{1}{2} q_{11} \lambda \bar{Z} \varepsilon_{1} \varepsilon_{2}+\left(\frac{3}{8} q_{9} \bar{Y} \lambda^{2}+\frac{1}{2} q_{10} \bar{X} \lambda\right) \varepsilon_{1}^{2} \\
& -\frac{1}{2} \psi \lambda\left(q_{10} \bar{X} C_{x}^{2}+q_{11} C_{x z} \bar{Z}+\frac{3}{4} q_{9} \bar{Y} \lambda C_{x}^{2}\right. \\
& \left.-q_{9} \bar{Y} C_{y x}\right) .
\end{aligned}
$$

We get approximately zero bias by taking expectation on both sides of Eq. (24). It is indicated that the proposed class generates Hartley-Ross type unbiased estimators.

$$
\operatorname{Bias}\left(\bar{y}_{P}^{(u)}\right)=E\left(\bar{y}_{P}^{(u)}-\bar{Y}\right) \cong 0 \text {. }
$$

To obtain the variance of the proposed estimators up to the first degree of approximation, both sides of Eq. (24) are squared and the expectation is taken as follows:

$$
\begin{aligned}
\operatorname{Var}\left(\bar{y}_{P}^{(u)}\right) & \cong \bar{Y}^{2} \psi C_{y}^{2}-\bar{Y}^{2} \psi \lambda V_{1} q_{9}-2 \bar{X} \bar{Y} \psi C_{y x} q_{10} \\
& -2 \bar{Y} \bar{Z} \psi C_{y z} q_{11}+\bar{Y}^{2} \lambda^{2} \psi V_{2} q_{9}^{2} \\
& +\bar{X}^{2} \psi C_{x}^{2} V_{3} q_{10}^{2}+\bar{Z}^{2} \psi C_{z}^{2} V_{4} q_{11}^{2} \\
& +\bar{X} \bar{Y} \psi \lambda C_{x} V_{5} q_{9} q_{10}+\bar{Y} \bar{Z} \psi \lambda V_{6} q_{9} q_{11} \\
& +2 \bar{X} \bar{Z} \psi C_{x z} V_{3} q_{10} q_{11}
\end{aligned}
$$

where:

$$
\begin{aligned}
V_{1}= & \frac{3}{4} \psi \lambda C_{y}^{2} C_{x}^{2}+C_{y x}-\frac{\psi C_{y x} C_{y} \theta_{21 x}}{\rho_{y x}}, \\
V_{2}= & \frac{1}{4} C_{x}^{2}-\frac{9}{64} \psi \lambda^{2} C_{x}^{4}-\frac{1}{4} \psi C_{y x}^{2}-\frac{\psi C_{y x} C_{x} \theta_{12 x}}{2 \rho_{y x}} \\
& +\frac{3}{4} \psi \lambda C_{x}^{2} C_{y x}, \\
V_{3}= & 1-\frac{1}{4} \psi \lambda^{2} C_{x}^{2}, \\
V_{4}= & 1-\frac{1}{4} \psi \lambda^{2} C_{x}^{2} \rho_{x z}^{2}, \\
V_{5}= & C_{x}+\frac{5}{4} \psi \lambda C_{x} C_{y x}-\frac{\psi C_{y x} \theta_{12 x}}{\rho_{y x}}-\frac{3}{8} \psi \lambda^{2} C_{x}^{3},
\end{aligned}
$$

$$
\begin{aligned}
V_{6}= & \frac{3}{4} \psi \lambda C_{x}^{2} C_{y z}+C_{x z}-\frac{\psi C_{y x} C_{z} \theta_{12 z}}{\rho_{y z}} \\
& +\frac{1}{2} \psi \lambda C_{x z} C_{y x}-\frac{3}{8} \psi \lambda^{2} C_{x}^{2} C_{x z} .
\end{aligned}
$$

In order to get the optimal values of $q_{i}^{\prime} s$, we differentiate Eq. (25) with respect to $q_{i}, i=9,10,11$, and then equate them to zero. Therefore, we get:

$$
\begin{aligned}
& q_{9(\mathrm{opt})}=\frac{2 B_{1}}{\lambda B_{2}}, \\
& q_{10(\mathrm{opt})}=\frac{\bar{Y}}{\bar{X}}\left[\frac{V_{1} A_{2} B_{2}-4 V_{2} A_{2} B_{1}-V_{6}\left(A_{3} B_{2}-A_{6} B_{1}\right)}{C_{x} V_{5} A_{2} B_{2}}\right], \\
& q_{11(\mathrm{opt})}=\frac{\bar{Y}}{\bar{Z}}\left[\frac{A_{3} B_{2}-A_{6} B_{1}}{A_{2} B_{2}}\right] .
\end{aligned}
$$

We obtained the minimum variance at optimal values of $q_{i}, i=9,10,11$, by inserting them in Eq. (25):

$$
\begin{aligned}
\operatorname{Var}_{\min }\left(\bar{y}_{P}^{(u)}\right) \cong \frac{1}{C_{x} V_{5}^{2} A_{2}^{2} B_{2}^{2}} \bar{Y}^{2} \psi\left[C _ { x } V _ { 5 } ^ { 2 } A _ { 2 } ^ { 2 } \left\{C_{y}^{2} B_{2}^{2}\right.\right. \\
\left.-2 V_{1} B_{1} B_{2}+4 V_{2} B_{1}^{2}\right\}+C_{1}\left\{C_{x} V_{3} C_{1}\right. \\
\left.-2 C_{y x} V_{5} A_{2} B_{2}+2 C_{x} V_{5}^{2} A_{2} B_{1}\right\} \\
+C_{x} V_{5}^{2} C_{2}\left\{C_{z}^{2} V_{4} C_{2}-2 C_{y z} A_{2} B_{2}\right. \\
\left.\left.+2 V_{6} A_{2} B_{1}\right\}+2 C_{x z} V_{3} V_{5} C_{1} C_{2}\right]
\end{aligned}
$$

where:

$$
\begin{aligned}
& A_{1}=C_{x} V_{1} V_{3}-C_{y x} V_{5} \\
& A_{2}=C_{x z} V_{3} V_{6}-C_{z}^{2} C_{x} V_{4} V_{5} \\
& A_{3}=C_{x z} V_{1} V_{3}-C_{y z} C_{x} V_{5} \\
& A_{4}=C_{x} V_{3} V_{6}-C_{x z} V_{3} V_{5} \\
& A_{5}=C_{x}\left(4 V_{2} V_{3}-V_{5}^{2}\right) \\
& A_{6}=4 C_{x z} V_{2} V_{3}-C_{x} V_{5} V_{6}, \\
& B_{1}=A_{1} A_{2}-A_{3} A_{4}, \quad B_{2}=A_{2} A_{5}-A_{4} A_{6} \\
& C_{1}=V_{1} A_{2} B_{2}-4 V_{2} A_{2} B_{1}-V_{6}\left(A_{3} B_{2}-A_{6} B_{1}\right) \\
& C_{2}=\left(A_{3} B_{2}-A_{6} B_{1}\right) .
\end{aligned}
$$




\section{Empirical illustration}

In this section, three natural populations are used to present the empirical performance of the suggested almost unbiased estimators as compared to other estimators. Table 3 contains helpful information regarding datasets. Percent Relative Efficiency (PRE) is calculated through the following expression:

$$
P R E=\frac{M S E\left(\bar{y}_{0}^{(u)}\right)}{M S E(\bullet)} \times 100,
$$

where:

$$
\begin{aligned}
\bullet= & \bar{y}_{0}^{(u)}, \bar{y}_{H R}^{(u)}, \bar{y}_{G K}, \bar{y}_{S 1}^{(u)}, \bar{y}_{S 2}^{(u)}, \bar{y}_{C K 1}^{(u)}, \bar{y}_{C K 2}^{(u)}, \bar{y}_{M U, 0}, \\
& \bar{y}_{M U, 1}, \bar{y}_{S G}, \bar{y}_{P}^{(u)} .
\end{aligned}
$$

We calculated the PREs of all the estimators taken from the literature and for the proposed family of estimators with respect to $\bar{y}_{0}^{(u)}$. Empirical findings are reported in Tables 4, 5, and 6 for Populations 1, 2, and 3 , respectively. From Tables 4, 5, and 6, the following observations are made:

- A comparison between all unbiased/Hartley-Rosstype unbiased estimators, i.e., $\bar{y}_{0}^{(u)}, \bar{y}_{H R}^{(u)}, \bar{y}_{S 1}^{(u)}, \bar{y}_{S 2}^{(u)}$, $\bar{y}_{C K 1}^{(u)}, \bar{y}_{C K 2}^{(u)}$, and $\bar{y}_{P}^{(u)}$ reveals that $\bar{y}_{P}^{(u)}$ provides maximum gain in PREs as compared to others. This gain in PREs is based on the fact that the proposed estimators use the information on two auxiliary variables, while the others use the information on only one auxiliary variable;

- The proposed family of estimators also provides greater PREs than the well-known Grover and Kaur [23] class of biased estimators, i.e., $\bar{y}_{G K}$. This class considers the information on only one auxiliary variable;

- When the proposed estimators $\bar{y}_{P}^{(u)}$ are contrasted against the biased estimators using the auxiliary information on two variables, i.e., $\bar{y}_{M U, 0}, \bar{y}_{M U, 1}$, $\bar{y}_{S G}$, we again observe the gain in PREs of the suggested estimators.

- Finally, it is quite obvious that the proposed family gives maximum PREs as compared to all other estimators under study.

Hence, the suggested family outperforms and provides almost unbiased and efficient estimators for estimating population mean in case of SRSWOR.

\section{Concluding remarks}

In this article, a new family of Hartley-Ross type unbiased estimators for estimating population mean under SRSWOR was suggested. In this family, the informa-

\begin{tabular}{|c|c|c|c|c|}
\hline & Population & 1 & 2 & 3 \\
\hline & \multirow{2}{*}{ Source } & \multicolumn{2}{|c|}{ Koyuncu and Kadilar [32] } & \multirow{2}{*}{$\begin{array}{c}\text { Singh and Mangat [22], } \\
\text { page } 369\end{array}$} \\
\hline & & Mediterranean region & Marmara region & \\
\hline \multirow{3}{*}{ 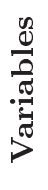 } & Study variable $y$ & Number of teachers & Number of teachers & Number of tube wells \\
\hline & Auxiliary variable $x$ & Number of classes & Number of classes & Number of tractors \\
\hline & Auxiliary variable $z$ & Number of students & Number of students & Net irrigated area \\
\hline \multirow{13}{*}{ 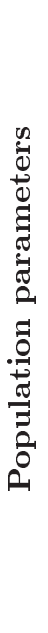 } & $N$ & 103 & 127 & 69 \\
\hline & $n$ & 29 & 31 & 10 \\
\hline & $\bar{Y}$ & 573.1748 & 703.74 & 135.2608 \\
\hline & $\bar{X}$ & 431.36 & 498.28 & 21.232 \\
\hline & $\bar{Z}$ & 14309.3 & 20804.59 & 345.7536 \\
\hline & $C_{y}$ & 1.8030 & 1.2559 & 0.8422 \\
\hline & $C_{x}$ & 1.4209 & 1.1150 & 0.7969 \\
\hline & $C_{z}$ & 1.9253 & 1.4654 & 0.8478 \\
\hline & $\rho_{y x}$ & 0.9835 & 0.9789 & 0.9119 \\
\hline & $\rho_{y z}$ & 0.9937 & 0.9366 & 0.9224 \\
\hline & $\rho_{x z}$ & 0.9765 & 0.9396 & 0.9007 \\
\hline & $\beta_{1(x)}$ & 3.1112 & 1.7205 & 1.8551 \\
\hline & $\beta_{2(x)}$ & 10.7864 & 2.3149 & 3.7653 \\
\hline
\end{tabular}
tion on two auxiliary variables was utilized to generate

Table 3. Data statistics. 
Table 4. PRE's of different estimators for Population 1.

\begin{tabular}{cccccccc}
\hline \multirow{2}{*}{ Estimator } & \multirow{2}{*}{ PREs } & \multicolumn{6}{c}{ Families of estimators } \\
\cline { 3 - 8 } & & $\boldsymbol{\alpha}$ & $\boldsymbol{\beta}$ & $\overline{\boldsymbol{y}}_{\boldsymbol{C K \mathbf { 1 }}}^{(\boldsymbol{u})}$ & $\overline{\boldsymbol{y}}_{\boldsymbol{C K \mathbf { 2 }}}^{(\boldsymbol{u})}$ & $\overline{\boldsymbol{y}}_{\boldsymbol{G} K}$ & $\overline{\boldsymbol{y}}_{\boldsymbol{P}}^{(\boldsymbol{u})}$ \\
\hline $\bar{y}_{0}^{(u)}$ & 100 & 1 & $C_{x}$ & 659.866 & 2443.415 & 3150.869 & $\mathbf{5 9 4 1 3 . 6 5 5}$ \\
$\bar{y}_{H R}^{(u)}$ & 1410.541 & 1 & $\beta_{2(x)}$ & 561.315 & 2491.637 & 3145.299 & $\mathbf{5 1 5 4 1 . 4 5 2}$ \\
$\bar{y}_{S 2}^{(u)}$ & 589.228 & $\beta_{2(x)}$ & $C_{x}$ & 678.997 & 2437.056 & 3151.680 & $\mathbf{6 0 7 3 6 . 1 1 0}$ \\
$\bar{y}_{S 1}^{(u)}$ & 670.420 & $\rho_{y x}$ & $C_{x}$ & 659.532 & 2443.533 & 3150.854 & $\mathbf{5 9 3 8 9 . 8 3 8}$ \\
$\bar{y}_{M U, 0}$ & 11417.693 & $\beta_{2(x)}$ & $\rho_{y x}$ & 679.634 & 2436.858 & 3151.705 & $\mathbf{6 0 7 7 8 . 7 8 6}$ \\
$\bar{y}_{M U, 1}$ & 11673.080 & $\rho_{y x}$ & $\beta_{2(x)}$ & 559.860 & 2492.604 & 3145.197 & $\mathbf{5 1 4 1 3 . 9 6 2}$ \\
$\bar{y}_{S G}$ & 12273.554 & 1 & $\rho_{y x}$ & 666.124 & 2441.249 & 3151.143 & $\mathbf{5 9 8 5 4 . 5 8 9}$ \\
& & $C_{x}$ & $\beta_{2(x)}$ & 589.228 & 2474.781 & 3147.139 & $\mathbf{5 3 9 3 0 . 5 4 1}$ \\
& & $C_{x}$ & $\rho_{y x}$ & 670.420 & 2439.813 & 3151.326 & $\mathbf{6 0 1 5 2 . 6 6 4}$ \\
& & 1 & $\beta_{1(x)}$ & 637.582 & 2451.854 & 3149.823 & $\mathbf{5 7 7 8 0 . 4 8 6}$ \\
& & $S_{x}$ & $\beta_{1(x)}$ & 680.996 & 2436.436 & 3151.760 & $\mathbf{6 0 8 6 9 . 8 2 2}$ \\
& 1 & $C_{z}$ & 652.919 & 2445.920 & 3150.555 & $\mathbf{5 8 9 1 4 . 8 0 9}$ \\
& & 1 & $\rho_{y z}$ & 665.975 & 2441.301 & 3151.137 & $\mathbf{5 9 8 4 4 . 1 9 2}$ \\
& & $\rho_{y z}$ & $C_{z}$ & 652.756 & 2445.981 & 3150.548 & $\mathbf{5 8 9 0 2 . 9 9 3}$ \\
\hline
\end{tabular}

Note: Bold values indicate maximum PREs.

Table 5. PREs of different estimators for Population 2.

\begin{tabular}{cccccccc}
\hline \multirow{2}{*}{ Estimator } & \multirow{2}{*}{ PREs } & \multicolumn{6}{c}{ Families of estimators } \\
\cline { 3 - 8 } & & $\boldsymbol{\alpha}$ & $\boldsymbol{\beta}$ & $\overline{\boldsymbol{y}}_{\boldsymbol{C} \mathbf{1} \mathbf{1}}^{(\boldsymbol{u})}$ & $\overline{\boldsymbol{y}}_{C K \mathbf{2}}^{(\boldsymbol{u})}$ & $\overline{\boldsymbol{y}}_{G K}$ & $\overline{\boldsymbol{y}}_{\boldsymbol{P}}^{(\boldsymbol{u})}$ \\
\hline $\bar{y}_{0}^{(u)}$ & 100 & 1 & $C_{x}$ & 1075.153 & 2489.092 & 2440.404 & $\mathbf{2 6 5 7 . 3 1 7}$ \\
$\bar{y}_{H R}^{(u)}$ & 1998.897 & 1 & $\beta_{2(x)}$ & 1042.644 & 2489.789 & 2440.104 & $\mathbf{2 6 5 6 . 7 5 5}$ \\
$\bar{y}_{S 2}^{(u)}$ & 1048.831 & $\beta_{2(x)}$ & $C_{x}$ & 1093.903 & 2488.732 & 2440.563 & $\mathbf{2 6 5 7 . 6 1 5}$ \\
$\bar{y}_{S 1}^{(u)}$ & 1082.030 & $\rho_{y x}$ & $C_{x}$ & 1074.465 & 2489.106 & 2440.398 & $\mathbf{2 6 5 7 . 3 0 5}$ \\
$\bar{y}_{M U, 0}$ & 1620.000 & $\beta_{2(x)}$ & $\rho_{y x}$ & 1095.707 & 2488.699 & 2440.578 & $\mathbf{2 6 5 7 . 6 4 3}$ \\
$\bar{y}_{M U, 1}$ & 1685.198 & $\rho_{y x}$ & $\beta_{2(x)}$ & 1041.368 & 2489.819 & 2440.092 & $\mathbf{2 6 5 6 . 7 3 1}$ \\
$\bar{y}_{S G}$ & 2591.975 & 1 & $\rho_{y x}$ & 1079.081 & 2489.014 & 2440.438 & $\mathbf{2 6 5 7 . 3 8 1}$ \\
& & $C_{x}$ & $\beta_{2(x)}$ & 1048.831 & 2489.649 & 2440.163 & $\mathbf{2 6 5 6 . 8 6 6}$ \\
& & $C_{x}$ & $\rho_{y x}$ & 1082.030 & 2488.957 & 2440.463 & $\mathbf{2 6 5 7 . 4 2 9}$ \\
& & 1 & $\beta_{1(x)}$ & 1058.295 & 2489.442 & 2440.252 & $\mathbf{2 6 5 7 . 0 3 3}$ \\
& & $S_{x}$ & $\beta_{1(x)}$ & 1108.930 & 2488.463 & 2440.684 & $\mathbf{2 6 5 7 . 8 4 2}$ \\
& & 1 & $C_{z}$ & 1065.279 & 2489.294 & 2440.316 & $\mathbf{2 6 5 7 . 1 5 2}$ \\
& & 1 & $\rho_{y z}$ & 1080.312 & 2488.990 & 2440.449 & $\mathbf{2 6 5 7 . 4 0 1}$ \\
& & $\rho_{y z}$ & $C_{z}$ & 1062.544 & 2489.351 & 2440.291 & $\mathbf{2 6 5 7 . 1 0 6}$ \\
\hline
\end{tabular}

Note: Bold values indicate maximum PREs.

new estimators. The mathematical expressions were derived for the Bias and the minimum variance of new family up to the first order of approximation. To evaluate the potentiality of the new family, three natural populations were used. Numerical findings confirmed the efficiency of new estimators as compared to:

i) The unbiased/Hartley-Ross-type unbiased estima- tors, for instance, traditional estimator, Hartley and Ross [12], Singh et al. [19] and Cekim and Kadilar [20];

ii) The biased estimators using the information of one or two auxiliary variable(s) such as Grover and Kaur [23], Muneer et al. [10], and Shabbir and Gupta [11]. 
Table 6. PREs of different estimators for Population 3.

\begin{tabular}{cccccccc}
\hline \multirow{2}{*}{ Estimator } & \multirow{2}{*}{ PREs } & \multicolumn{6}{c}{ Families of estimators } \\
\cline { 3 - 7 } & & $\boldsymbol{\alpha}$ & $\boldsymbol{\beta}$ & $\overline{\boldsymbol{y}}_{\boldsymbol{C K \mathbf { 1 }}}^{(\boldsymbol{u})}$ & $\overline{\boldsymbol{y}}_{\boldsymbol{C K \boldsymbol { 2 }}}^{(\boldsymbol{u})}$ & $\overline{\boldsymbol{y}}_{G K}$ & $\overline{\boldsymbol{y}}_{\boldsymbol{P}}^{(\boldsymbol{u})}$ \\
\hline $\bar{y}_{0}^{(u)}$ & 100 & 1 & $C_{x}$ & 576.732 & 533.718 & 609.601 & $\mathbf{9 2 4 . 4 2 8}$ \\
$\bar{y}_{H R}^{(u)}$ & 589.345 & 1 & $\beta_{2(x)}$ & 548.203 & 532.040 & 606.910 & $\mathbf{9 1 0 . 8 4 3}$ \\
$\bar{y}_{S 2}^{(u)}$ & 515.877 & $\beta_{2(x)}$ & $C_{x}$ & 539.836 & 534.694 & 610.309 & $\mathbf{9 2 7 . 9 5 2}$ \\
$\bar{y}_{S 1}^{(u)}$ & 587.853 & $\rho_{y x}$ & $C_{x}$ & 579.800 & 533.609 & 609.513 & $\mathbf{9 2 3 . 9 8 9}$ \\
$\bar{y}_{M U, 0}$ & 872.685 & $\beta_{2(x)}$ & $\rho_{y x}$ & 587.853 & 533.261 & 609.214 & $\mathbf{9 2 2 . 4 9 3}$ \\
$\bar{y}_{M U, 1}$ & 899.339 & $\rho_{y x}$ & $\beta_{2(x)}$ & 536.079 & 532.110 & 606.657 & $\mathbf{9 0 9 . 5 5 3}$ \\
$\bar{y}_{S G}$ & 898.269 & 1 & $\rho_{y x}$ & 581.180 & 533.557 & 609.471 & $\mathbf{9 2 3 . 7 7 5}$ \\
& & $C_{x}$ & $\beta_{2(x)}$ & 515.877 & 532.319 & 606.272 & $\mathbf{9 0 7 . 5 7 9}$ \\
& & $C_{x}$ & $\rho_{y x}$ & 587.852 & 533.261 & 609.214 & $\mathbf{9 2 2 . 4 9 3}$ \\
& & 1 & $\beta_{1(x)}$ & 592.953 & 532.574 & 608.490 & $\mathbf{9 1 8 . 8 5 7}$ \\
& & $S_{x}$ & $\beta_{1(x)}$ & 530.559 & 534.893 & 610.440 & $\mathbf{9 2 8 . 6 0 4}$ \\
& & 1 & $C_{z}$ & 578.804 & 533.646 & 609.543 & $\mathbf{9 2 4 . 1 3 7}$ \\
& & 1 & $\rho_{y z}$ & 581.547 & 533.543 & 609.459 & $\mathbf{9 2 3 . 7 1 6}$ \\
& & $\rho_{y z}$ & $C_{z}$ & 581.437 & 533.547 & 609.462 & $\mathbf{9 2 3 . 7 3 3}$ \\
\hline
\end{tabular}

Note: Bold values indicate maximum PREs.

On the basis of these findings, it can be recommended using the new family for future applications.

\section{Acknowledgments}

The authors would like to express their heartfelt thanks to the learned referees for their constructive comments and suggestions that led to this improved version of the article and to the Editor-in-Chief, Prof. S.T.A. Niaki.

\section{References}

1. Abu-Dayyeh, W.A., Ahmed, M.S., Ahmed, R.A., and Muttlak, H.A. "Some estimators of finite population mean using auxiliary information", Applied Mathematics and Computation, 139, pp. 287-298 (2003).

2. Kadilar, C. and Cingi, H. "A new estimator using two auxiliary variables", Applied Mathematics and Computation, 162, pp. 901-908 (2005).

3. Singh, H.P. and Tailor, R. "Estimation of finite population mean using known correlation coefficient between auxiliary characters", Statistica, 65, pp. 407418 (2005).

4. Lu, J. and Yan, Z. "A class of ratio estimators of a finite population mean using two auxiliary variables", PLOS ONE, 9(2), pp. 1-6 (2014).

5. Lu, J., Yan, Z., and Peng, X. "A new exponential ratio type estimator with linear combination of two auxiliary variables", PLOS ONE, 9(12), pp. 1-10 (2014).

6. Vishwakarma., G.K. and Kumar, M. "A general family of dual to ratio-cum-product estimators of population mean in simple random sampling", Chilean Journal of Statistics, 6(2), pp. 69-79 (2015).
7. Sharma, P. and Singh, R. "A class of exponential ratio estimators of finite population mean using two auxiliary variables", Pakistan Journal of Statistics and Operational Research, 11(2), pp. 221-229 (2015).

8. Yasmeen, U., Amin, N.M., and Hanif, M. "Exponential ratio and product type estimators of finite population mean", Journal of Statistics and Management Systems, 19(1), pp. 55-71 (2016).

9. Lu, J. "Efficient estimator of a finite population mean using two auxiliary variables and numerical application in agriculture, biomedical and power engineering", Mathematical Problems in Engineering, Article ID 8704734 (2017). https://doi.org $/ 10.1155 / 2017 / 8704734$

10. Muneer, S., Shabbir, J., and Khalil, A. "Estimation of finite population mean in simple random sampling and stratified random sampling using two auxiliary variables", Communications in Statistics- Theory and Methods, 46(5), pp. 2181-2192 (2017).

11. Shabbir, J. and Gupta, S. "Estimation of finite population mean in simple and stratified random sampling using two auxiliary variables", Communications in Statistics- Theory and Methods, 46(20), pp. 1013510148 (2017).

12. Hartley, H.O. and Ross, A. "Unbiased ratio estimators", Nature, 174, pp. 270-272 (1954).

13. Robson, D.S. "Application of multivariate polykays to the theory of unbiased ratio type estimation", Journal of American Statistical Association, 50, pp. 1225-1226 (1957).

14. Murthy, M.N. and Nanjamma, N.S. "Almost unbiased estimator based on interpenetrating sub-sample estimates", Sankhya, 21, pp. 381-392 (1959). 
15. Biradar, R.S. and Singh, H.P. "A note on almost unbiased ratio-cum-product estimator", Metron, $40(1-$ 2), pp. 249-255 (1992).

16. Biradar, R.S. and Singh, H.P. "On a class of almost unbiased ratio estimators", Biomedical Journal, 34(8), pp. 937-944 (1992).

17. Biradar, R.S. and Singh, H.P. "A class of unbiased ratio estimators", Journal of Indian Society Agricultural Statistics, 47(3), pp. 230-239 (1995).

18. Sahoo, J., Sahoo, L.N., and Mohanty, S. "An alternative approach to estimation in two phase sampling using two auxiliary variables", Biometrical Journal, 36, pp. 293-298 (1994).

19. Singh, H.P., Sharma, B., and Tailor, R. "HartleyRoss type estimators for population mean using known parameters of auxiliary variate", Communications in Statistics- Theory and Methods, 43, pp. 547-565 (2014).

20. Cekim, H.O. and Kadilar, C. "New unbiased estimators with the help of Hartley-Ross type estimators", Pakistan Journal of Statistics, 32(4), pp. 247-260 (2016).

21. Khan, L., Shabbir, J., and Gupta, S. "Unbiased ratio estimators of the mean in stratified ranked set sampling", Hacettepe Journal of Mathematics and Statistics, 46(6), pp. 1151-1158 (2017).

22. Singh, R. and Mangat, N.S., Elements of Survey Sampling, Norwell, MA: Kluwer Academic Publishers (1996).

23. Grover, L.K. and Kaur, P. "A generalized class of ratio type exponential estimators of population mean under linear transformation of auxiliary variable", Communications in Statistics-Simulation and Computation, 43, pp. 1552-1574 (2014).

24. Gupta, S. and Shabbir, J. "On improvement in estimating the population mean in simple random sampling", Journal of Applied Statistics, 35(5), pp. 559566 (2008).

25. Shabbir, J. and Gupta, S. "On estimating finite population mean in simple and stratified random sampling", Communications in Statistics- Theory and Methods, 40(2), pp. 199-212 (2011).

26. Kadilar, C. and Cingi, H., A New Ratio Estimator Using Correlation Coefficient, Inter-Stat, pp. 1-11 (2006).

27. Upadhyaya, L.N. and Singh, H.P. "Use of transformed auxiliary variable in estimating the finite population mean", Biometrical Journal, 41(5), pp. 627-636 (1999).
28. Khoshnevisan, M., Singh, R., Chauhan, P., Sawan, N., and Smarandache, F. "A general family of estimators for estimating population mean using known value of some population parameter(s)", Far East Journal of Theoretical Statistics, 22, pp. 181-191 (2007).

29. Koyuncu, N. and Kadilar, C. "Efficient estimators for the population mean", Hacettepe Journal of Mathematics and Statistics, 38(2), pp. 217-225 (2009).

30. Singh, V.K. and Singh, R. "Performance of an estimator for estimating population mean using simple and stratified random sampling", SOP Transactions on Statistics and Analysis, 1(1), pp. 1-8 (2014).

31. Grover, L.K. and Kaur, P. "An improved estimator of the finite population mean in simple random sampling", Model Assisted Statistics and Application, 6(1), pp. 47-55 (2011).

32. Koyuncu, N. and Kadilar, C. "Family of estimators of population mean using two auxiliary variables in stratified sampling", Communications in StatisticsTheory and Methods, 38, pp. 2398-2417 (2009).

\section{Biographies}

Maria Javed is currently a PhD scholar in Statistics at Zhejiang University, Hangzhou, China. She received her MPhil degree in Statistics from Government College University, Faisalabad, Pakistan. She has been working as a Lecturer at the Department of Statistics, Government College University, Faisalabad, Pakistan since 2004. Her research interests include sampling theory and probability distributions.

Muhammad Irfan obtained his PhD degree in Statistics from Zhejiang University, Hangzhou, China in 2018. He has held the position of a Lecturer at the Department of Statistics, Government College University, Faisalabad, Pakistan since 2004. He has more than 15 research publications in well-reputed journals. His areas of interest are sampling theory, probability distributions, and time series analysis.

Tianxiao Pang is an Associate Professor of Statistics at Zhejiang University, Hangzhou, China. He has about 30 refereed publications now and has supervised research students at all levels of the curriculum including undergraduate and $\mathrm{PhD}$ students. 